\title{
Segmentation of Images Containing Multiple Intensity Levels using Genetic Algorithms
}

\author{
B.D. Phulpagar, $\mathrm{PhD}$ \\ Computer Engineering Department \\ P. E. S. Modern College of Engineering, \\ Pune, India.
}

\author{
R.S. Bichkar, PhD \\ Electronics and Telecommunication Department \\ G. H. Raisoni College of Engineering and \\ Management, Pune, India.
}

\begin{abstract}
Image segmentation technique is the process of separating the foreground objects of different intensities from the background. Several authors have proposed different methods for segmentation of images into two classes, each one having different quality of segmentation. Yu et al [1]. used a GA approach to segmentation of 2-D images into two classes. We have extended this method to segment the images into multiple classes or multiple intensity levels (Four, Eight, Twelve and Sixteen greay Levels). The proposed GA-based approach gives us good results for original synthetic images and noisy images containing rectangular, elliptical and irregular-objects using morphological operations upto sixteen classes. The results obtained give $81 \%$ to $100 \%$ pixel classification accuracy for different types of noise (Gaussian, Speckle, salt and pepper and Poisson) and high noise levels (SNR ranging between $2.18 \mathrm{~dB}$ to $6.97 \mathrm{~dB}$ ). The segmentation results obtained by using proposed GA-based method are good as compared to standard image segmentation FCM method with the increasing noise density of salt and pepper and Gaussian noises.
\end{abstract}

\section{General Terms}

Image Processing, Pattern Recognition.

\section{Keywords}

Genetic Algorithm, Multiple Intensity Levels, Image Segmentation.

\section{INTRODUCTION}

Genetic Algorithm is an optimization technique that is inspired by the Darwinian theory of evolution and is mainly based on the principle of survival of the fittest. It was introduced by John Holland to simulate the genetic systems. GA was used by Goldberg [2] for the optimization of pipelines. He has presented a detailed mathematical model of a GA. Initially its results are based on the principles of natural selection and set of populations. It does not require domain specific knowledge in their search method. In every generation of GA, the generation of a new population of candidate solution depends on genetic operators like reproduction, crossover and mutation. GA have been used to solve the problems in computer vision area like image segmentation, image enhancement, image compression, watermarking, steganography, object recognition and feature selection.

The initial attempts for GA-based segmentation of images separated objects of one intensity from uniform background. This required a single optimal threshold value to be determined. It has been suggested in many cases that the single threshold methods can be extended to multiplethresholding for more than two-class problems. The segmentation of images into multiple classes has also been attempted by using region and cluster based segmentation techniques. Zhang and Ciesielski [3] have presented GAbased multiple class object detection technique to find out the locations of small objects of multiple classes in large pictures. The limitation of the proposed method is that it classifies the images upto three classes. Chabrier et al. [4] have proposed an optimization based image segmentation by using GA. The criterion is optimized by using genetic algorithm. Nath et al. [5] have proposed a histogram-based method for image segmentation by incorporating multi-level thresholding and a hill climbing algorithm. A GA-based technique to find the near-optimal threshold has been proposed by Banimelhem and Yahya [6]. The proposed technique segments the images into three classes. The results of this technique have been presented for images containing no noise. Kumar et al. [7] have presented an automatic multi-level thresholding method for image segmentation using discrete wavelet transforms (DWT) and genetic algorithm. P. Liao et al. [8], Samanta et al. [9] and Rawi et al. [10] have also proposed GA-based methods for segmentation of images into two or more classes.

The limitation of these existing methods motivated us to explore the GA-based image segmentation for images containing objects of different shapes and intensities in presence of noise.

In this paper, the GA-based method is implemented for reconstruction of images containing rectangular, elliptical and irregular shaped objects is considered. In order to evolve individual images containing rectangular, elliptical and irregular shaped objects, selected a random morphological operator among the considered set of morphological operators and also select structuring element at random among the provided set of structuring elements for noisy input images upto sixteen classes. The results obtained using GA-based method show an improvement in classification accuracy as compared to standard image segmentation FCM method.

\section{IMAGE SEGMENTATION USING GA}

The objective of the proposed GA-based technique for image segmentation is to separate the rectangular, elliptical and irregular-shaped objects from the background. The images are considered to comprise of four, eight, twelve or sixteen constant greay levels and corrupted by different types of noises (salt and pepper, Gaussian, Speckle and Poisson). Consider an input image of size $m \times n$. In this method, segmentation is performed on $4 \times 4$ or $8 \times 8$ non-overlapping sub-images, which are later combined to obtain an entire segmented image. GA is an efficient population-based search and global optimization method. The length of the chromosomes $(L)$ is fixed. It is equals to the number of pixels in a sub-image. A chromosome in GA represents a segmented 
2-D image. The structure of the chromosome is a vector representing 2-D sub-image of size $4 \times 4$ or $8 \times 8$. For an image containing $\mathrm{C}$ classes, each gene of chromosomes can have upto $\mathrm{C}$ possible values: $(0,1,2, \ldots, \mathrm{C}-1)$. Each individual has a fitness value that is some measure of its closeness to the actual solution.

The implementation of GA is similar to that in [11] except that all the operations have been modified to handle multiple intensity levels. Thus, the initial population of $\mathrm{GA}$ is randomly generated. The set of individuals of the first generation represents the initial population in the search space. The fitness function is used to establish a link between the GA and application problem to be solved. It returns a value associated with each chromosome indicating how fit it is in a given environment. To measure the fitness of an image the fitness function is used as explained in [11]. In this proposed method, we have used roulette wheel selection [2] to select the parents in reproduction step.

Reproduction step generates the population of qualified individuals for the next generation. In this method, it consists of three functions i.e. morphological operations, crossover and mutation. The results of multiple-class images with morphological operations are obtained by extending the basic binary morphological operations [12], [13]. In dilation, the image pixel is replaced by the maximum of the pixels considered by the structuring element. Thus it brightens and expands brighter areas of an image and darkens and shrinks darker areas. In erosion, the image pixel is replaced by minimum of the pixels considered by the structuring element. Thus it is the dual of the dilation and has the opposite effect. For multi-class images, the opening and closing operations have the same form as that in a binary image. Thus multi-class opening is multi-class erosion followed by multi-class dilation and multi-class closing is multi-class dilation followed by multi-class erosion. The effect of closing on multi-class images is that the small objects brighter than background and preserved and bright objects with small gaps in between may become connected. On the other hand in opening operation, removes bright objects that are small in size and breaks narrow connections between two bright objects

To identify rectangular, elliptical or irregular objects contained in an image, we have used morphological operators, selected at random among the following set: opening followed by closing, closing followed by opening, opening, closing, dilation and erosion to identify approximate irregular shaped objects containing in individual images. The structuring element $(Q)$ is a $3 \times 3$ mask with an appropriate intensity structure.

The crossover operator [2] creates two new offsprings from two parent strings by swapping their parts about a randomly selected crossover point with crossover probability $p_{c}=0.60$.

The mutation operator flips the bits of chromosomes with small probability $p_{m}=0.06$. Generally, offsprings are mutated after they are created during the of crossover operator. It improves the fitness of an individual. The elitist method is incorporated to increase diversity in populations. The program is terminated when a specified number of generations are completed.

\section{RESULTS AND DISCUSSIONS}

In this work, an attempt has been made to separate the foreground objects of different intensities from the background by using genetic algorithm. The input images contain upto sixteen greay levels (four, eight, twelve and sixteen greay levels). Also, the effect of image block sizes and noise is considered. The number of objects in test image and their shapes are evolved. To speed up the GA run, the image is divided into non-overlapping sub-images of size $4 \times 4$ or $8 \times 8$ pixels. Later segmented sub-images are combined to obtain the entire segmented image. Here, the chromosomes size is an array of 16 or 64 integer values. To understand the effect of noise, four types of noise are considered. These include salt and pepper, Gaussian, Speckle and Poisson.

The noisy images are obtained from images shown in Figure 1 (a). The salt and pepper noise is added with noise density of 0.04 and 0.08 to this original image to obtain noisy images with SNR of $63.25 \mathrm{~dB}$ and $24.70 \mathrm{~dB}$ as shown in Figure 2 (a) and (b). Also, Figure 2 (c) is obtained by adding Poisson noise with $6.97 \mathrm{~dB}$ SNR and Figure 2 (d) - (f) are noisy images with $3.63 \mathrm{~dB}, 2.66 \mathrm{~dB}, 2.41 \mathrm{~dB}$ SNR obtained by adding Speckle noise with density $0.02,0.04$ and 0.08 , Figure $2(\mathrm{~g})-$ (h) are noisy images with $2.65 \mathrm{~dB}$ and $2.18 \mathrm{~dB}$ SNR obtained by adding Gaussian noise with density 0.02 and 0.04 respectively.

The GA parameters are constant for all the experiments i.e. mutation probability $=0.06$, crossover probability $=0.60$ and seed value $=1$. The population size and generations vary as shown in Table 1 and Table 2. A morphological operator is selected at random among set of morphological operators.

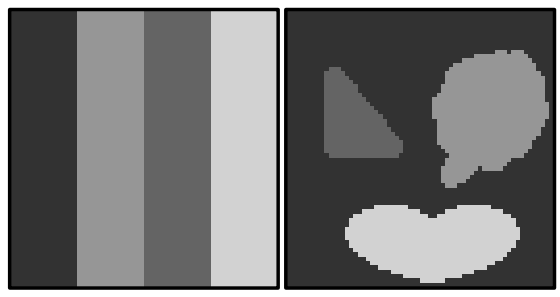

(a)

(b)

Figure 1: Set of synthetic images.

Figure 3 shows the results obtained using proposed GA for noisy test images shown in Figure 2. It may be observed that very good segmentation is obtained with pixel classification accuracy of $100 \%$ for both salt and pepper noise (Figure 3 (a) and (b)) and Poisson noise (Figure 3 (c)). The results in Figure 3 (d) - (f) and (g) - (h) are for images having Speckle noise and Gaussian noise, the segmentation error for which is less than $14 \%$ and $19 \%$ respectively. Table 1 shows the overall summary for image segmentation of images shown in Figure 3 using GA with one-point crossover operators. It is observed that for images having SNR more than $3.00 \mathrm{~dB}$ the classification accuracy is excellent (almost 100\%). Also observe that the images required larger population size and more GA generations for higher noise density levels. The above experiment was repeated with a larger image block size of $8 \times 8$ and the results obtained are shown in Figure 4 and Table 2. It may be observed that very good segmentation is obtained with pixel classification accuracy of $100 \%$ and $95 \%$ for salt and pepper noise (Figure 4 (a) - (b)) and Poisson noise (Figure 4 (c)) respectively. Figure 4 (d) - (f) and (g) - (h) show segmentation results for images having Speckle and Gaussian noise for which the segmentation error is less than $14 \%$ and $16 \%$ respectively. Table 2 summarizes the results shown in Figure 4. 


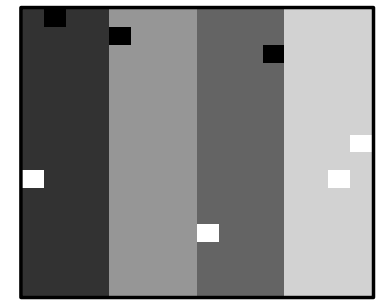

(a)

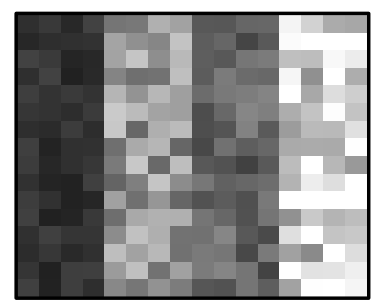

(e)

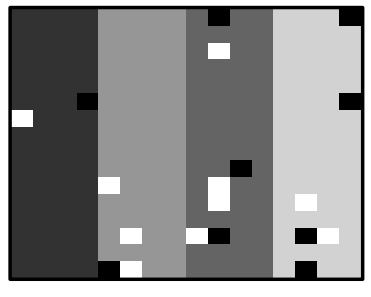

(b)

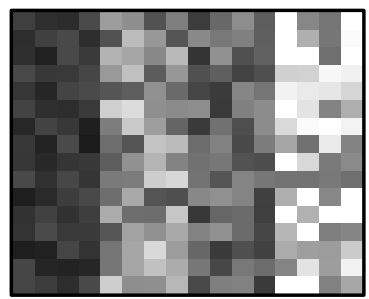

(f)

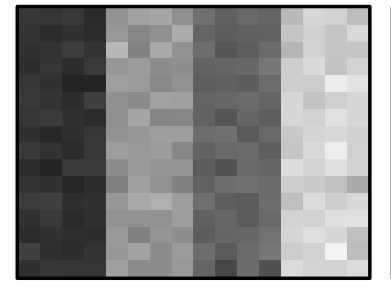

(c)

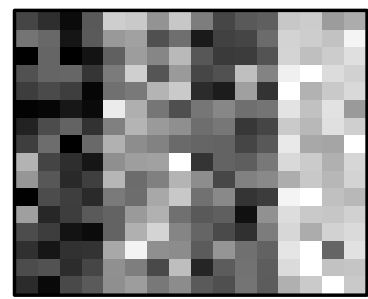

(g)

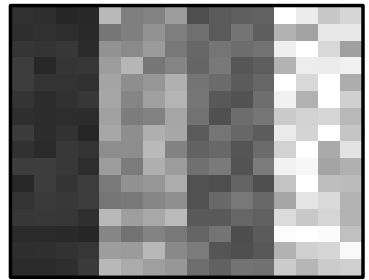

(d)

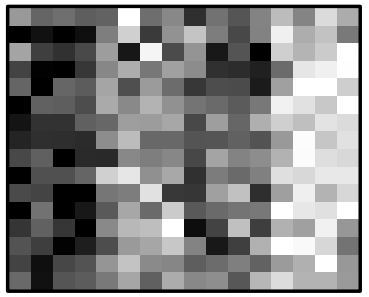

(h)

Figure 2: Noisy image set obtained by adding different types of noise to image shown in Figure 1 (a) having four classes. Images (a) - (b) contain salt and pepper noise with 63.25 dB and 24.70 dB SNR, (c) Noisy images with Poisson noise with 6.97 dB SNR, (d) - (f) Noisy images with Speckle noise with 3.63 dB, $2.66 \mathrm{~dB}$ and $2.41 \mathrm{~dB}$ SNR, (g) - (h) Noisy images with Gaussian noise with SNR of $2.65 \mathrm{~dB}$ and $2.18 \mathrm{~dB}$.

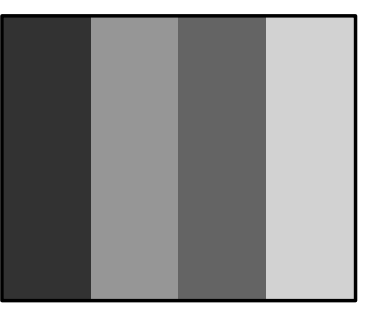

(a)

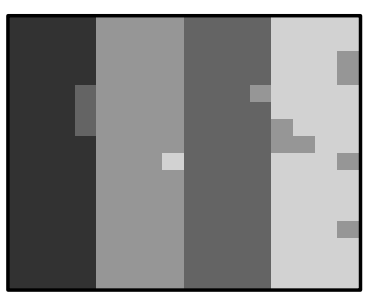

(e)

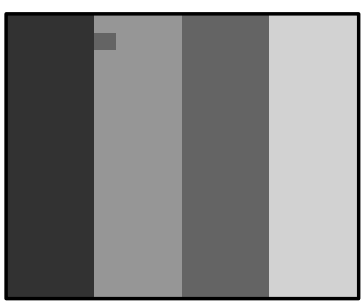

(b)

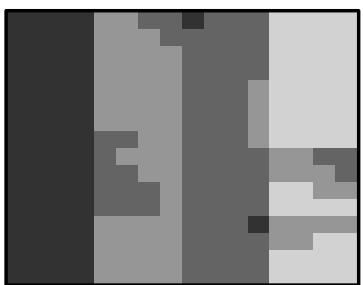

(f)

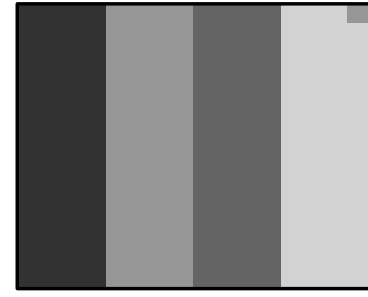

(c)

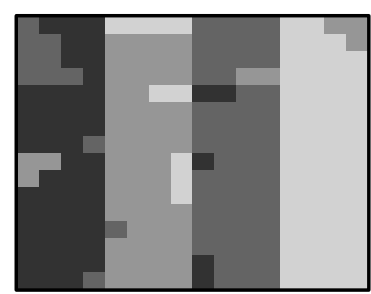

(g)

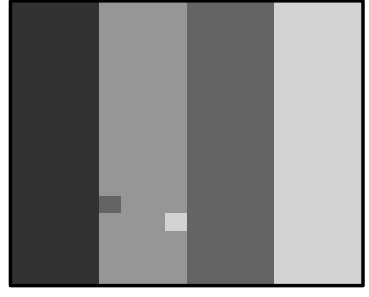

(d)

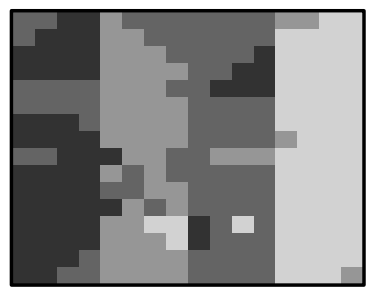

(h)

Figure 3: Segmentation results obtained with image block size $4 \times 4$ pixels by using proposed GA for noisy image set shown in Figure 2.

Table 1: Summary of segmentation results shown in Figure 3 for four class noisy images obtained using proposed GA-based approach with image block size $4 \times 4$ pixels.

\begin{tabular}{|c|c|c|c|c|c|c|c|c|}
\hline Sr. No. & $\begin{array}{l}\text { Noisy } \\
\text { Image }\end{array}$ & $\begin{array}{c}\text { Types of } \\
\text { Noise }\end{array}$ & $\begin{array}{c}\text { Noise } \\
\text { Density }\end{array}$ & $\begin{array}{c}\text { Image } \\
\text { SNR } \\
\text { in dB }\end{array}$ & $\begin{array}{c}\text { Population } \\
\text { size }\end{array}$ & $\begin{array}{c}\text { GA } \\
\text { Generations }\end{array}$ & $\begin{array}{l}\text { Error in } \\
\text { Pixels }\end{array}$ & $\begin{array}{c}\text { Accuracy } \\
\text { in } \%\end{array}$ \\
\hline \multirow{2}{*}{1} & \multirow{2}{*}{$\begin{array}{r}\text { Figure } 2 \\
\text { (a) }-(b)\end{array}$} & \multirow{2}{*}{$\begin{array}{l}\text { salt and } \\
\text { pepper }\end{array}$} & 0.04 & 63.25 & 10 & 28 & 1 & 100 \\
\hline & & & 0.08 & 24.70 & 12 & 32 & 1 & 100 \\
\hline 2 & Figure 2 (c) & Poisson & -- & 6.97 & 10 & 30 & 1 & 100 \\
\hline \multirow{3}{*}{3} & \multirow{3}{*}{$\begin{array}{l}\text { Figure } 2 \\
\text { (d) }-(\mathrm{f})\end{array}$} & \multirow{3}{*}{ Speckle } & 0.02 & 3.63 & 12 & 36 & 2 & 99 \\
\hline & & & 0.04 & 2.66 & 16 & 38 & 12 & 95 \\
\hline & & & 0.08 & 2.41 & 18 & 40 & 36 & 86 \\
\hline \multirow{2}{*}{4} & \multirow{2}{*}{$\begin{array}{l}\text { Figure } 2 \\
(\mathrm{~g})-(\mathrm{h})\end{array}$} & \multirow{2}{*}{ Gaussian } & 0.02 & 2.65 & 16 & 40 & 33 & 87 \\
\hline & & & 0.04 & 2.18 & 22 & 44 & 50 & 81 \\
\hline
\end{tabular}


It may be observed that the use of $8 \times 8$ block size requires larger population size and more number of GA generations to achieve the results obtained by GA with $4 \times 4$ image block size. Thus, the $4 \times 4$ image block size is more suitable for segmentation of such multi-class images.

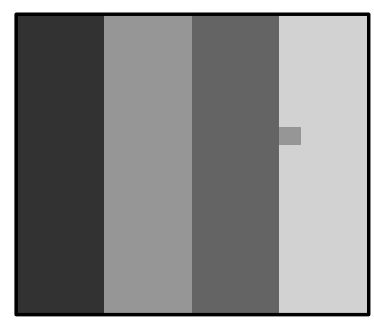

(a)

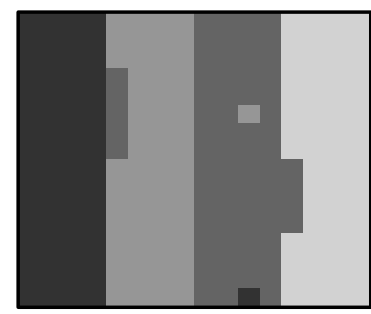

(e)

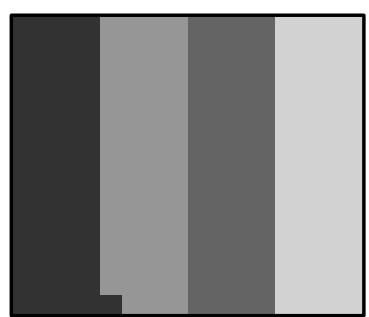

(b)

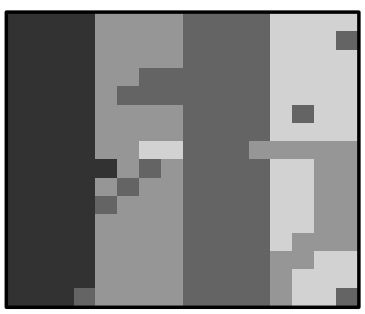

(f)

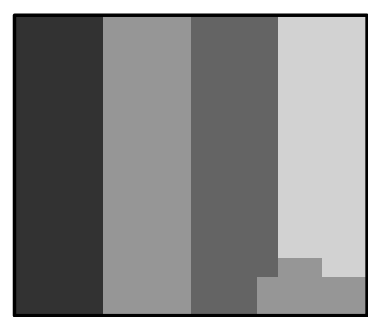

(c)

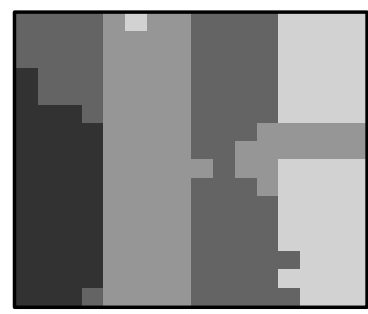

(g)

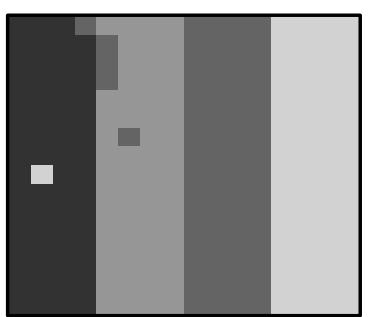

(d)

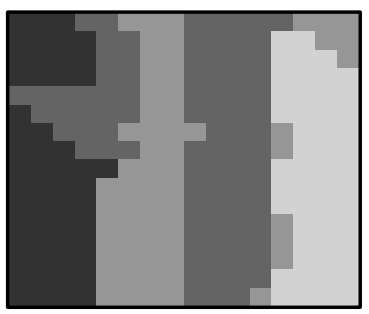

(h)

Figure 4: Segmentation results obtained with block size $8 \times 8$ pixels by using proposed GA for noisy image set shown in Figure 2.

Table 2: Summary of segmentation results shown in Figure 4 for four classes using proposed GA-based approach with image block size $8 \times 8$ pixels. $\%$ Segmentation accuracy in percentage

\begin{tabular}{|c|c|c|c|c|c|c|c|c|}
\hline Sr. No. & $\begin{array}{l}\text { Noisy } \\
\text { Image }\end{array}$ & $\begin{array}{c}\text { Type of } \\
\text { Noise }\end{array}$ & $\begin{array}{c}\text { Noise } \\
\text { Density }\end{array}$ & $\begin{array}{c}\text { Image } \\
\text { SNR } \\
\text { in dB }\end{array}$ & $\begin{array}{c}\text { Population } \\
\text { size }\end{array}$ & $\begin{array}{c}\text { GA } \\
\text { Generations }\end{array}$ & $\begin{array}{l}\text { Error in } \\
\text { Pixels }\end{array}$ & $\begin{array}{c}\text { Accuracy } \\
\text { in } \%\end{array}$ \\
\hline \multirow{2}{*}{1} & \multirow{2}{*}{$\begin{array}{l}\text { Figure } 2 \\
\text { (a) }-(b)\end{array}$} & \multirow{2}{*}{$\begin{array}{l}\text { salt and } \\
\text { pepper }\end{array}$} & 0.04 & 63.25 & 22 & 300 & 1 & 100 \\
\hline & & & 0.08 & 24.70 & 22 & 310 & 1 & 100 \\
\hline 2 & Figure 2(c) & Poisson & -- & 6.97 & 20 & 200 & 12 & 95 \\
\hline \multirow{3}{*}{3} & \multirow{3}{*}{$\begin{array}{l}\text { Figure } 2 \\
(\mathrm{~d})-(\mathrm{f})\end{array}$} & \multirow{3}{*}{ Speckle } & 0.02 & 3.63 & 22 & 325 & 6 & 98 \\
\hline & & & 0.04 & 2.66 & 26 & 320 & 11 & 96 \\
\hline & & & 0.08 & 2.41 & 26 & 275 & 35 & 86 \\
\hline \multirow{2}{*}{4} & Figure 2 & \multirow{2}{*}{ Gaussian } & 0.02 & 2.65 & 26 & 380 & 38 & 85 \\
\hline & $(g)-(h)$ & & 0.04 & 2.18 & 28 & 405 & 40 & 84 \\
\hline
\end{tabular}

The proposed technique is next applied for segmentation of four class images containing elliptical and irregular-shaped objects shown in Figure 1 (b). The noisy images obtained from these images are shown in Figure 5. The salt and pepper noise is added with noise density level of 0.02, 0.04 and 0.08 to the original image Figure 1 (b) to obtain Figure 5 (a) - (c) with $106 \mathrm{~dB}, 47.65 \mathrm{~dB}$ and $4.0 \mathrm{~dB}$ SNR. Figure 5 (d) - (f) show the noisy images obtained by adding Gaussian noise with density $0.02,0.04$ and 0.08 to Figure 1 (b) with SNR of $2.38 \mathrm{~dB}, 2.04 \mathrm{~dB}$ and $1.83 \mathrm{~dB}$ respectively. The GA parameters are the same as used in the previous experiment. However, the population size and number of generations vary as shown in Table 3.

Figure 6 (a) - (f) shows the results obtained using proposed GA-based method for noisy test images shown in Figure 5. It may be observed that error is high in segmented results for salt and pepper and Gaussian noisy images with density level more than 0.04. Table 3 shows the overall summary of segmentation of images into four classes using proposed GAbased method for images shown in Figure 6. 


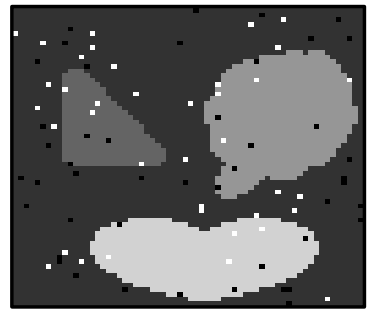

(a)

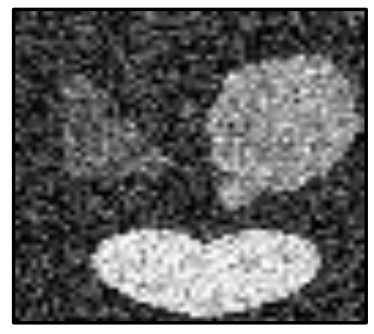

(d)

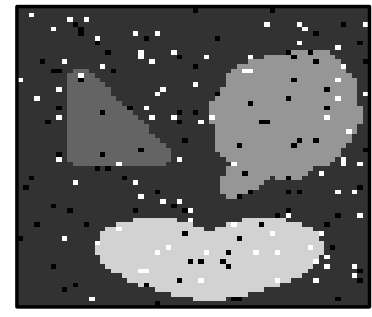

(b)

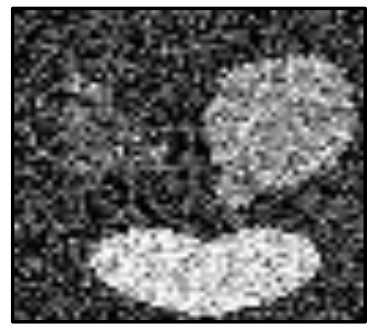

(e)

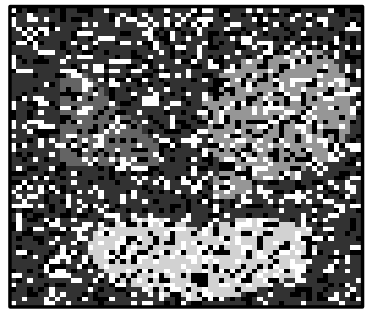

(c)

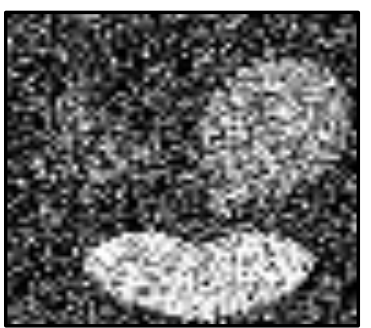

(f)

Figure 5: Noisy image set obtained by adding different types of noise to image shown in Figure 1 (b). (a) - (c) are noisy images containing salt and pepper noise with $106 \mathrm{~dB}, 47.65 \mathrm{~dB}$ and 4.0 dB SNR, and (d) - (f) are noisy images containing Gaussian noise with $2.38 \mathrm{~dB}, 2.04 \mathrm{~dB}$ and $1.83 \mathrm{~dB}$ SNR.

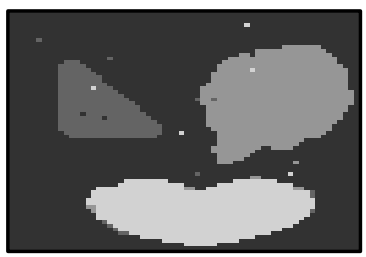

(a)

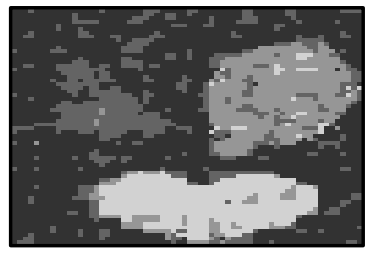

(d)

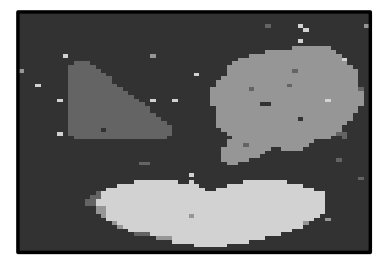

(b)

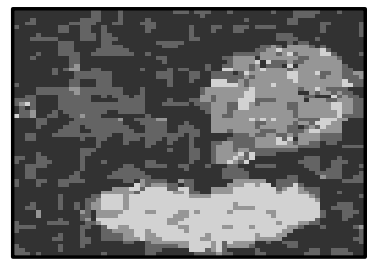

(e)

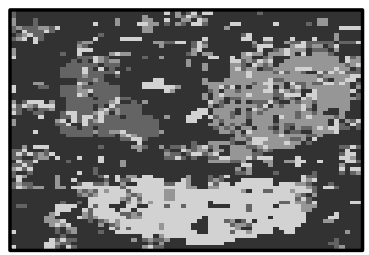

(c)

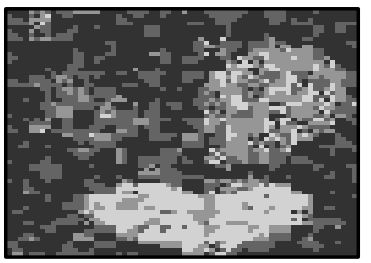

(f)

Figure 6: Segmentation results obtained with block size $4 \times 4$ pixels by using proposed GA for noisy image set shown in Figure 5.

Table 3: Summary of segmentation of images into four greay classes using GA-based method with image block size of $4 \times 4$ pixels for images shown in Figure 6.

\begin{tabular}{|c|c|c|c|c|c|c|c|c|}
\hline Sr. No. & $\begin{array}{l}\text { Noisy } \\
\text { Image }\end{array}$ & $\begin{array}{l}\text { Type of } \\
\text { Noise }\end{array}$ & $\begin{array}{c}\text { Noise } \\
\text { Density }\end{array}$ & $\begin{array}{c}\text { Image } \\
\text { SNR } \\
\text { in dB }\end{array}$ & $\begin{array}{l}\text { Population } \\
\text { size }\end{array}$ & $\begin{array}{c}\text { GA } \\
\text { Generations }\end{array}$ & $\begin{array}{c}\text { Error in } \\
\text { Pixels }\end{array}$ & $\begin{array}{c}\text { Error in } \\
\%\end{array}$ \\
\hline \multirow{3}{*}{1} & \multirow{3}{*}{$\begin{array}{l}\text { Figure } 5 \\
\text { (a) - (c) }\end{array}$} & \multirow{3}{*}{$\begin{array}{l}\text { Salt and } \\
\text { Pepper }\end{array}$} & 0.02 & 106.0 & 60 & 340 & 43 & 1 \\
\hline & & & 0.04 & 47.65 & 60 & 350 & 65 & 2 \\
\hline & & & 0.08 & 4.0 & 60 & 350 & 974 & 23 \\
\hline \multirow{3}{*}{2} & \multirow{3}{*}{$\begin{array}{l}\text { Figure } 5 \\
\text { (d) }- \text { (f) }\end{array}$} & \multirow{3}{*}{ Gaussian } & 0.02 & 2.38 & 10 & 50 & 693 & 16 \\
\hline & & & 0.04 & 2.04 & 12 & 46 & 1117 & 27 \\
\hline & & & 0.08 & 1.83 & 12 & 46 & 1457 & 35 \\
\hline
\end{tabular}


Next, we compare the results obtained by the proposed method with fuzzy c-means (FCM) method: Figure 7 shows the results obtained by using fuzzy c-means (FCM) method. It may be observed that the quality of image segmentation decreases with the increasing noise density of salt and pepper and Gaussian noises. It is also observed that the segmentation results obtained by using proposed GA-based method shown in Figure 6 are better as compared to segmentation results obtained by using FCM method shown in Figure 7. Table 4 shows a comparison of these results.

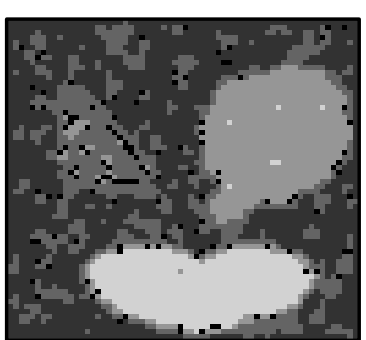

(a)

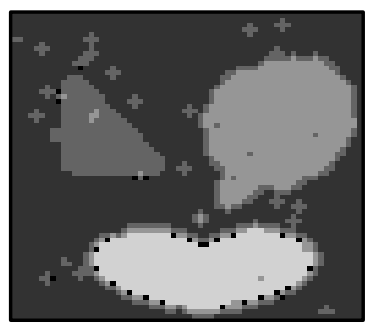

(d)

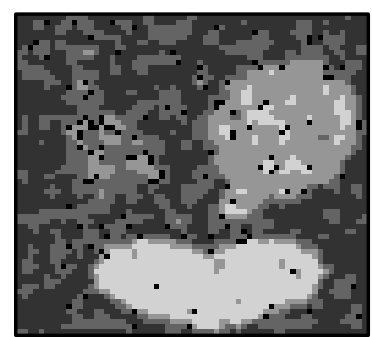

(b)

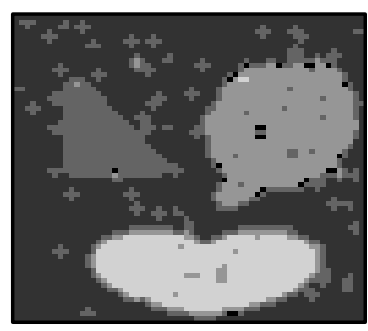

(e)

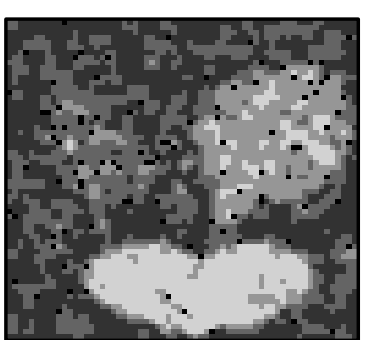

(c)

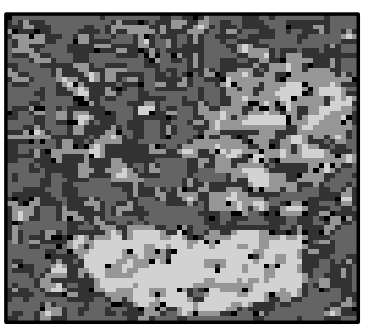

(f)

Figure 7: Segmentation results obtained by using FCM method for noisy images shown in Figure 5.

Table 4: Segmentation results obtained by using FCM technique and proposed GA-based method for noisy images shown in Figure 5.

\begin{tabular}{|c|c|c|c|c|c|c|}
\hline \multirow{2}{*}{ Sr. No. } & \multirow{2}{*}{$\begin{array}{l}\text { Noisy } \\
\text { Image }\end{array}$} & \multirow{2}{*}{$\begin{array}{c}\text { Type of } \\
\text { Noise }\end{array}$} & \multirow{2}{*}{$\begin{array}{c}\text { Noise } \\
\text { Density }\end{array}$} & \multirow{2}{*}{$\begin{array}{c}\text { Image } \\
\text { SNR in dB }\end{array}$} & \multicolumn{2}{|c|}{ Misclassified pixels } \\
\hline & & & & & FCM & GA \\
\hline \multirow{3}{*}{1} & \multirow{3}{*}{$\begin{array}{l}\text { Figure } 5 \\
(\mathrm{a})-(\mathrm{c})\end{array}$} & \multirow{3}{*}{$\begin{array}{c}\text { Salt and } \\
\text { Pepper }\end{array}$} & 0.02 & 106.0 & 414 & 43 \\
\hline & & & 0.04 & 47.65 & 540 & 65 \\
\hline & & & 0.08 & 4.0 & 2344 & 974 \\
\hline \multirow{3}{*}{2} & \multirow{3}{*}{$\begin{array}{l}\text { Figure } 5 \\
\text { (d) - (f) }\end{array}$} & \multirow{3}{*}{ Gaussian } & 0.02 & 2.38 & 986 & 693 \\
\hline & & & 0.04 & 2.04 & 1379 & 1117 \\
\hline & & & 0.08 & 1.83 & 1513 & 1457 \\
\hline
\end{tabular}

Next, the proposed technique is applied for segmentation of eight, twelve and sixteen class images containing rectangular, circular, elliptical and irregular-shaped objects as shown in Figure 8. The segmentation of image into eight or more classes is very difficult. This problem is overcome by using proposed method with larger population sizes and number of generations. It also works very well with the noisy images. For the experimentation, we have considered synthetic images shown in Figure 8 of $32 \times 32,64 \times 64$ and $128 \times 128$ pixels with eight, twelve and sixteen classes.

Figure 9 shows the segmentation results for the images shown in Figure 8. The segmentation accuracy is $100 \%$ with images containing rectangular objects and $98 \%$ with images containing circular, elliptical and irregular-shaped objects. To achieve $100 \%$ segmentation accuracy with image block size of $8 \times 8$ GA required larger population size and number of generations. 


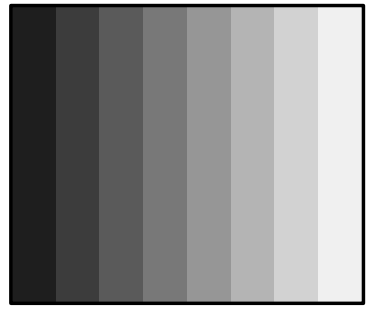

(a)

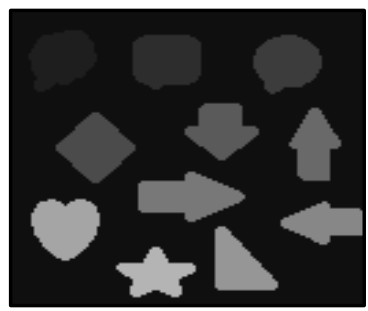

(d)

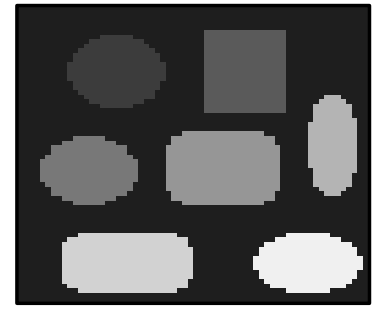

(b)

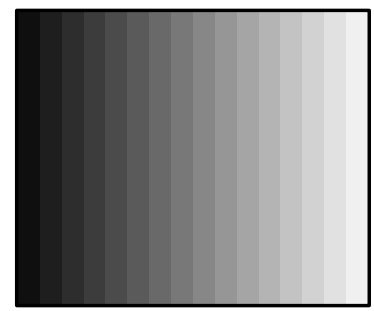

(e)

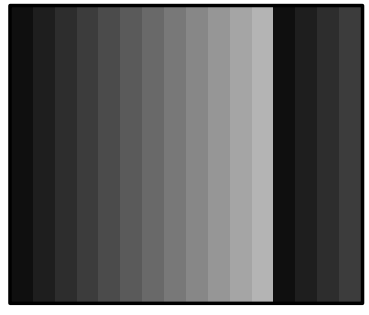

(c)

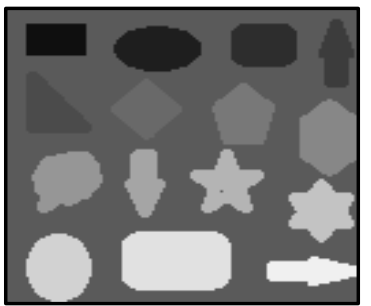

(f)

Figure 8: Synthetic images with eight $(a-b)$, twelve $(c-d)$ and sixteen $(e-f)$ classes respectively.

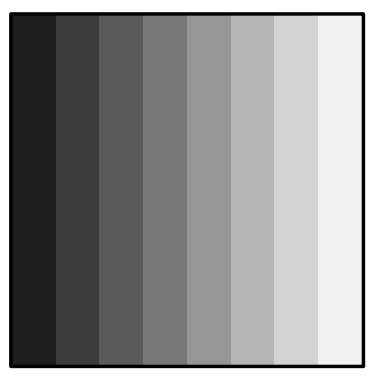

(a)

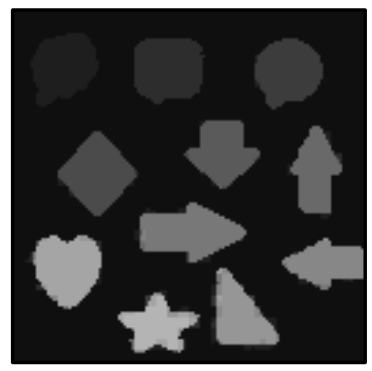

(d)

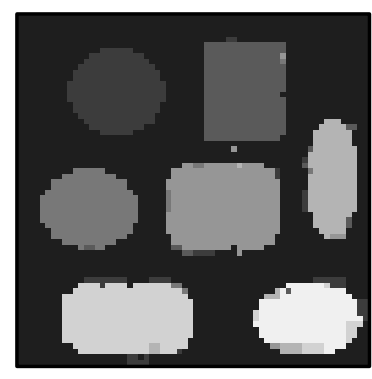

(b)

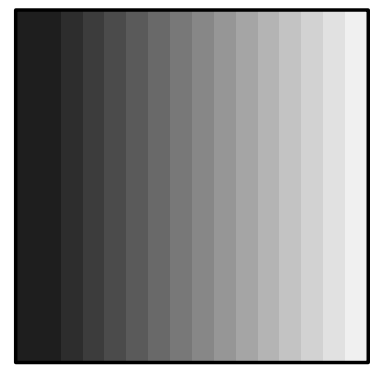

(e)

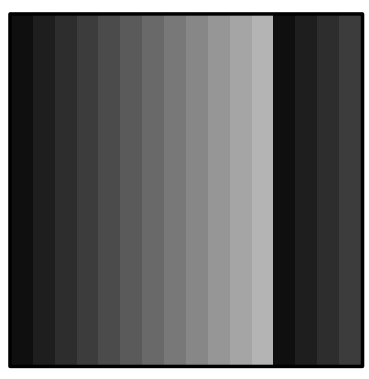

(c)

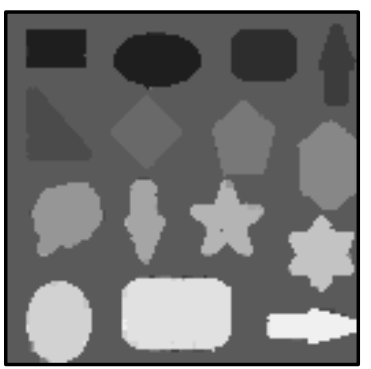

(f)

Figure 9: Segmentation of images into eight $(a-b)$, twelve $(c-d)$ and sixteen $(e-f)$ classes by using proposed GA-based method with image block size of $4 \times 4$ pixels.

To analyze the effectiveness of proposed GA-based method for segmentation of multiple class images, several noisy images are obtained from the images shown in Figure 8. They are shown in Figure 10. Figure 10 (a) is obtained from Figure 8 (a) by adding Poisson noise and has $5.65 \mathrm{~dB}$ SNR. Figure 10 (b) - (d) are images obtained from image in Figure 8 (a) by adding Speckle noise with density $0.02,0.04$ and 0.08 . These images have SNR of $3.46 \mathrm{~dB}, 2.87 \mathrm{~dB}$ and $2.85 \mathrm{~dB}$. Similarly, the salt and pepper noise is added with noise density level of $0.02,0.04$ and 0.08 to the image shown in Figure 8 (c) to obtain noisy images shown Figure 10 (e) - (g) with SNR of $95.35 \mathrm{~dB}, 52.88 \mathrm{~dB}$ and $20.56 \mathrm{~dB}$, Figure 10 (h) is also a noisy image with $90.35 \mathrm{~dB}$ SNR containing salt and pepper noise obtained by adding noise of density 0.02 to Figure 8 (e). The GA parameters are same as in the previous experiment but larger population sizes and more-number generations are used here.

Figure 11 shows the results obtained using proposed GAbased method for noisy test images shown in Figure 10. It may be observed that the results are good for segmentation of images into eight classes with pixel classification accuracy of $96 \%$ and $87 \%$ for Poisson and Speckle noises respectively as shown in Figure 11 (a) and (b). However, in case of Speckle noise with higher noise density level the quality of segmentation decreases as shown in Figure 11 (c) and (d) respectively. Figure 11 (e) - (g) shows segmentation of images into twelve classes and the segmentation error is less than $3 \%$ for images having salt and pepper noise. Figure 11 (h) shows the segmented image into sixteen classes with 279 


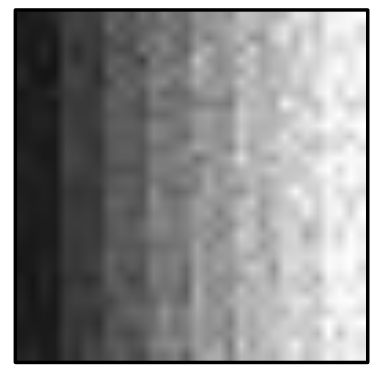

(a)

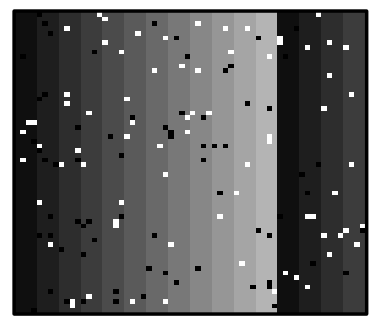

(e)

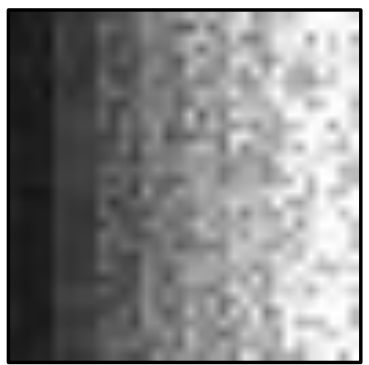

(b)

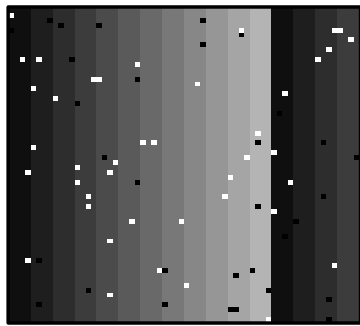

(f)

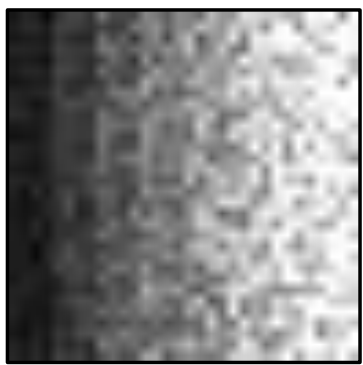

(c)

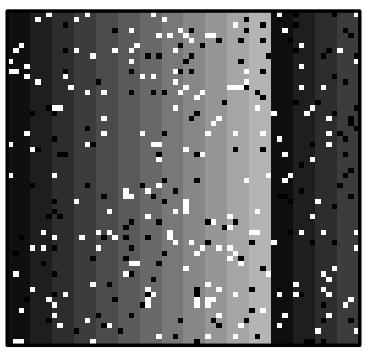

(g)

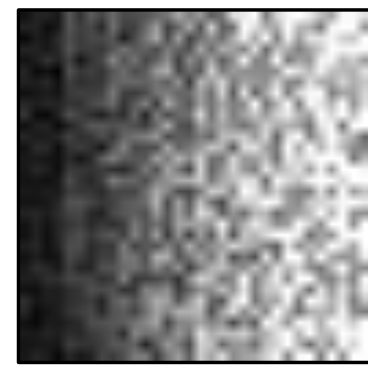

(d)

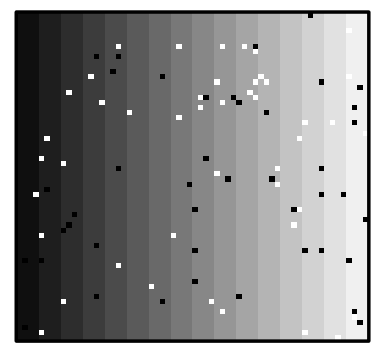

(h)

Figure 10: Noisy image set obtained by adding different types of noise to images shown in Figure 8 (a), (c) and (e) having eight, twelve and sixteen classes. (a) Poisson noise (5.65 dB SNR), (b) - (d) Speckle noise (3.46 dB, $2.87 \mathrm{~dB}$ and $2.85 \mathrm{~dB}$ SNR), (e) - (h) salt and pepper noise $(95.35 \mathrm{~dB}, 52.88 \mathrm{~dB}, 20.56 \mathrm{~dB}$ and $90.35 \mathrm{~dB}$ SNR) respectively.

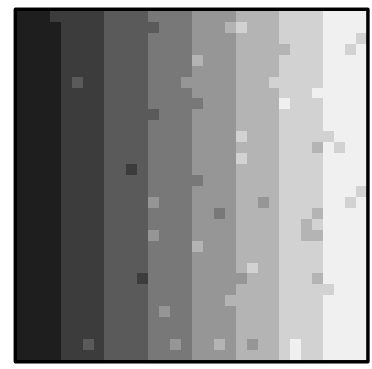

(a)

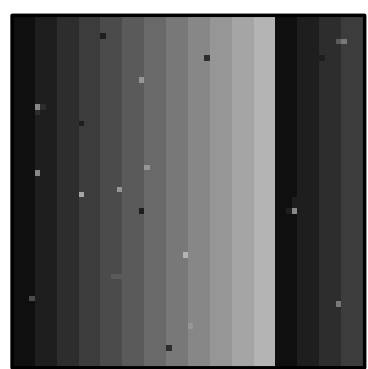

(e)

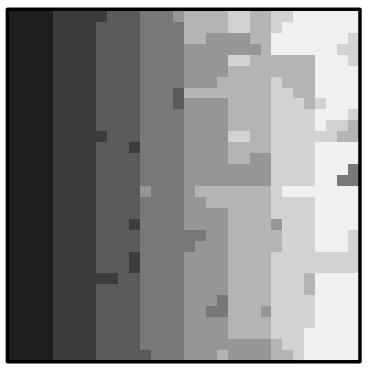

(b)

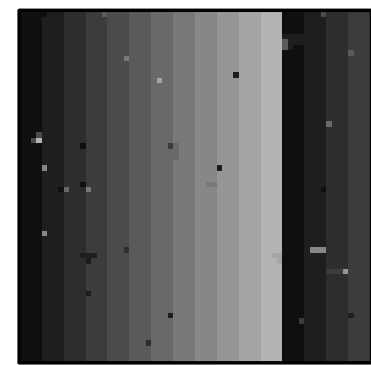

(f)

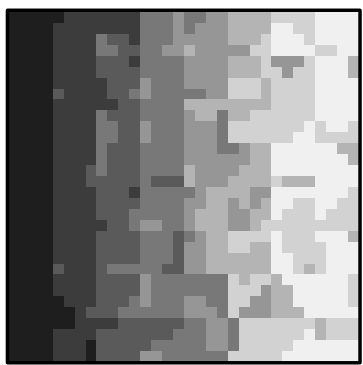

(c)

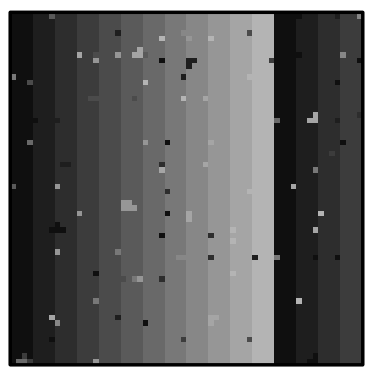

(g)

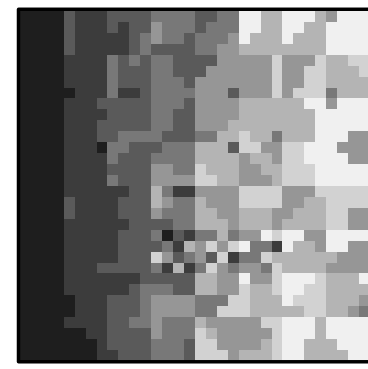

(d)

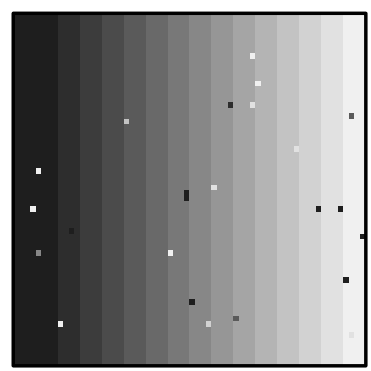

(h)

Figure 11: Segmentation of images into eight, twelve and sixteen classes obtained by using proposed GA for noisy images shown in Figure 10.

Table 5 Summaries the results obtained by using proposed GA for segmentation of images into eight, twelve and sixteen classes shown in Figure 11. It is observed that noisy images with low SNR (less than $3.00 \mathrm{~dB}$ ) required larger population size and more number of generations and the complexity of image segmentation increases with greay levels as well as increase in noise density levels. 
Table 5: Summary of segmentation of images into eight, twelve and sixteen greay classes using GA-based method for output images shown in Figure 11.

\begin{tabular}{|c|c|c|c|c|c|c|c|c|}
\hline Sr. No. & Noisy Image & $\begin{array}{c}\text { Type of } \\
\text { Noise }\end{array}$ & $\begin{array}{l}\text { Greay } \\
\text { Levels }\end{array}$ & $\begin{array}{c}\text { Image } \\
\text { SNR } \\
\text { in dB }\end{array}$ & $\begin{array}{c}\text { Population } \\
\text { size }\end{array}$ & $\begin{array}{c}\text { GA } \\
\text { Generations }\end{array}$ & $\begin{array}{c}\text { Error in } \\
\text { Pixels }\end{array}$ & $\underset{\%}{\text { Accuracy }}$ \\
\hline 1 & $\begin{array}{c}\text { Figure } 10 \text { (a) } \\
(32 \times 32)\end{array}$ & Poisson & \multirow{4}{*}{8} & 5.65 & 18 & 60 & 46 & 96 \\
\hline \multirow{3}{*}{2} & Figure 10 & \multirow{3}{*}{ Speckle } & & 3.46 & 32 & 123 & 139 & 87 \\
\hline & (b) $-(\mathrm{d})$ & & & 2.87 & 42 & 141 & 321 & 69 \\
\hline & $(32 \times 32)$ & & & 2.85 & 48 & 146 & 456 & 56 \\
\hline \multirow{3}{*}{3} & Figure 10 & \multirow{3}{*}{$\begin{array}{l}\text { salt and } \\
\text { pepper }\end{array}$} & \multirow{3}{*}{12} & 95.35 & 20 & 180 & 26 & 99 \\
\hline & (e) - (g) & & & 52.88 & 20 & 180 & 56 & 99 \\
\hline & $(64 \times 64)$ & & & 20.56 & 30 & 200 & 116 & 97 \\
\hline 4 & $\begin{array}{c}\text { Figure } 10 \\
\text { (h) } \\
(64 \times 64)\end{array}$ & $\begin{array}{l}\text { salt and } \\
\text { pepper }\end{array}$ & 16 & 90.35 & 40 & 200 & 279 & 93 \\
\hline
\end{tabular}

The proposed tchnique is also applied for segmentation of eight and twelve class images containing circular, elliptical and irregular-shaped objects shown in Figure 8 (b) and (d). Here, we have considered synthetic image of size $64 \times 64$ or $128 \times 128$. The noisy images obtained from these images are shown in Figure 12. The Speckle noise is added with noise density level of $0.02,0.04$ and 0.08 to image Figure 8 (b) to obtain Figure 12 (a) - (c) with $6.57 \mathrm{~dB}, 5.32 \mathrm{~dB}$ and $3.95 \mathrm{~dB}$ SNR. The noisy images shown in Figure 12 (d) and (e) are obtained by adding salt and pepper noise with density 0.04 and 0.08 to Figure 8 (d) with SNR of $40.73 \mathrm{~dB}$ and $3.69 \mathrm{~dB}$ respectively. The GA parameters are same as used in the previous experiments. The GA runs required larger population size and more number generations to identify the objects in these images.

Figure 13 shows the results obtained using proposed GAbased method for noisy test images shown in Figure 12. It may be observed that error is high in segmented results for images containing Speckle and salt and pepper noise with density level more than 0.04 . Table 6 shows the overall summary of segmented images into eight and twelve classes using proposed GA-based method for images shown in Figure 13.

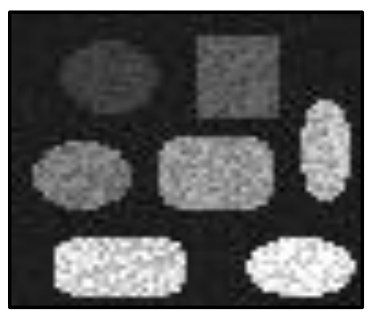

(a)

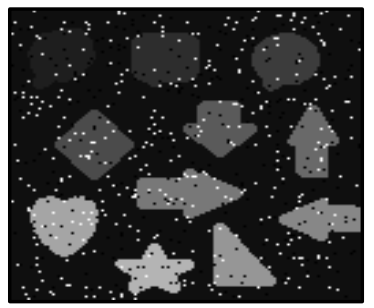

(d)

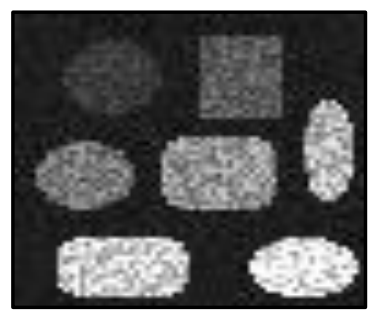

(b)

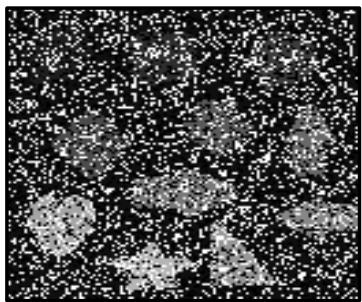

(e)

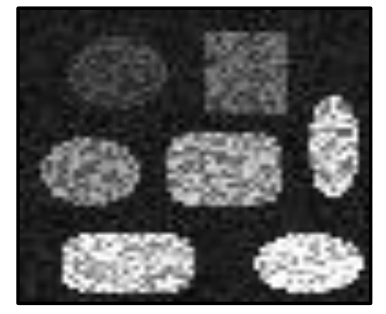

(c)

Figure 12: Noisy image set obtained by adding different types of noise to images shown in Figure 8 (b) and (d) having eight and twelve classes. (a) - (c) Speckle noise (6.57 dB, 5.32 dB, 3.95 dB SNR) and (d) - (e) salt and pepper noise (40.73 dB and 3.69 dB SNR). 


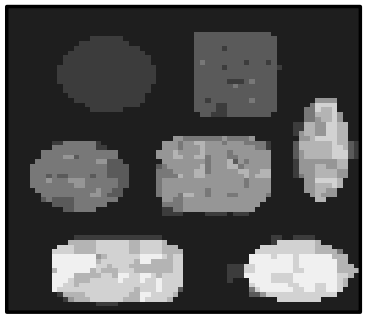

(a)

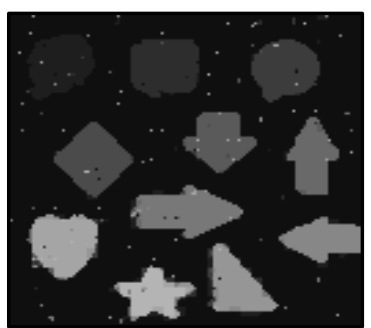

(d)

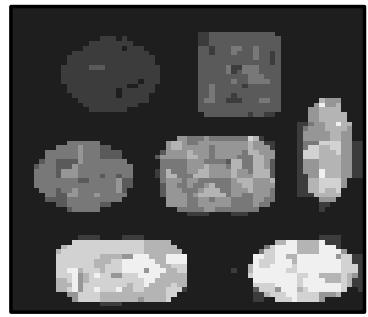

(b)

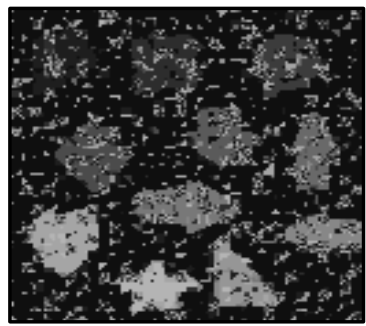

(e)

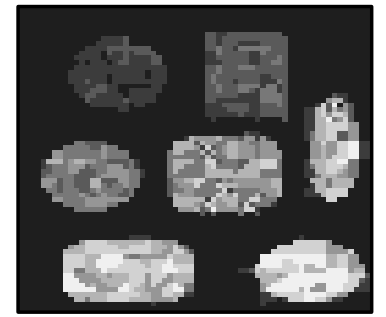

(c)

Figure 13: Segmentation of images into eight and twelve classes obtained by using proposed GA for noisy images shown in Figure 12.

Table 6: Summary of segmentation of images into eight and twelve greay classes using GA-based method for output images shown in Figure 13.

\begin{tabular}{|c|c|c|c|c|c|c|c|c|}
\hline \multirow{2}{*}{ Sr. No. } & $\begin{array}{c}\text { Noisy } \\
\text { Image }\end{array}$ & $\begin{array}{c}\text { Noise } \\
\text { Type }\end{array}$ & $\begin{array}{c}\text { Greay } \\
\text { Levels }\end{array}$ & $\begin{array}{c}\text { Image } \\
\text { SNR } \\
\text { in dB }\end{array}$ & $\begin{array}{c}\text { Population } \\
\text { size }\end{array}$ & $\begin{array}{c}\text { GA } \\
\text { Generations }\end{array}$ & $\begin{array}{c}\text { Error in } \\
\text { Pixels }\end{array}$ & $\begin{array}{c}\text { Accuracy } \\
\text { \% }\end{array}$ \\
\hline \multirow{2}{*}{1} & $\begin{array}{c}\text { Figure 12 } \\
(\mathrm{a})-(\mathrm{c}) \\
(64 \times 64)\end{array}$ & Speckle & 8 & 5.32 & 90 & 180 & 748 & 82 \\
\hline & $\begin{array}{c}\text { Figure 12 } \\
(\mathrm{d})-(\mathrm{e}) \\
(128 \times 128)\end{array}$ & $\begin{array}{c}\text { Salt and } \\
\text { pepper }\end{array}$ & \multirow{2}{*}{12} & 3.95 & 120 & 180 & 1026 & 75 \\
\cline { 5 - 10 } & & & 40.73 & 124 & 135 & 680 & 96 \\
\hline
\end{tabular}

\section{CONCLUSION}

A GA has been proposed for the image segmentation of 2-D images more than two classes corrupted with Poisson, salt and pepper, Gaussian and Speckle type of noises. The proposed method gives good results for the images containing rectangular, circular, elliptical and irregular shaped objects corrupted with Poisson, salt and pepper, Gaussian and Speckle noises with varying densities. The proposed method that the use of $8 \times 8$ block size requires larger population size and more number of GA generations to achieve the best results as compared to results obtained by GA with $4 \times 4$ image block size. The segmentation results are improved by $2 \%$ to $10 \%$ using proposed GA-based method as compared to standard image segmentation FCM method with the increasing noise density level of Gaussian and Speckle noises. Finally to achieve good segmentation results required larger population size and more number of generations with increases in greay levels as well as increase in noise density levels.

\section{REFERENCES}

[1] M. Yu, N. Eua-anant, A. Saudagar, and L. Udpa, "Genetic Algorithm Approach to Image Segmentation Using Morphological Operations," IEEE International Conference on Image Processing, vol. 3, pp. 775 - 779, October - 1998.
[2] D. E. Goldberg, Genetic Algorithm in Search, Optimization and Machine Learning, Pearson, Delhi, $2004,7^{\text {th }}$ Edition.

[3] M. Zhang and V. Ciesielski, "Genetic Programming for Multiple Class Object Detection," Proceeding of the 12th Australian Joint Conference on Artificial Intelligence Springer, Heidelberg, pp. 180 - 191, 1999.

[4] S. Chabrier, C. Rosenberger, and B. Emile, "Optimization Based Image Segmentation by Genetic Algorithms," EURASIP Journal on Video and Image Processing, vol. 1, pp. 1 - 23, February - 2008.

[5] S. Nath, S. Agarwal, and Q. Kazmi, "Image Histogram Segmentation by Multi-Level Thresholding using Hill Climbing Algorithm," IJCA, vol. 35, no. 1, pp. 63 - 72 , December - 2011.

[6] O. Banimelhem and Y. Yahya, "Multi-Thresholding Image Segmentation using Genetic Algorithm," Jordan University of Science and Technology, , Irbid, Jordan, pp. 1 - 6.

[7] R. Kumar, T. Parashar, and G. Verma, "A Multilevel Automatic Thresholding for Image Segmentation using 
Genetic Algorithm and DWT," International Journal of Electronics and Computer Science Engineering , vol. 1, no. 1 , pp. 153 - 160, 2013.

[8] P. Liao, T. Chen, and P. Chung, "A Fast Algorithm for Multilevel Thresholding," Journal of Information Science and Engineering, pp. 712 - 727, 2001.

[9] S. Samanta, N. Dey, and S. Acharjee, "Multilevel Threshold Based Greay Scale Image Segmentation using Cuckoo Search," Elsevier, pp. 27 - 34 , 2012.

[10] H. Rawi, Dr. Jane, and J. Stephan, "Histogram-Based Optimal Multiple Thresholding using Genetic Algorithm," University of Bahrain, pp. 1 - 8

[11] B. D. Phulpagar and R. S. Bichkar, "Segmentation of Noisy Binary Images Containing Circular and Elliptical Objects using Genetic Algorithms", International Journal of Computer Applications, vol. 66, no. 22, PP. 1 - 7, March - 2013.

[12] R. C. Gonzalez, R. E. Woods, Digital Image Processing, Pearson, Delhi, 2008, $3{ }^{\text {rd }}$ Edition.

[13] A. K. Jain, Fundamental of Digital Image Processing, Pearson, Delhi, 1989, $2^{\text {nd }}$ Edition.
[14] E. Gose, R. C. Johnsonbaugh, Pattern Recognition and Image Analysis, PrenticeHall, Delhi, 2000, $2{ }^{\text {nd }}$ Edition.

\section{AUTHOR PROFILE}

Dr. Phulpagar B. D. is currently working as Professor in Computer Engineering Department at P. E. S. Modern College of Engineering Pune (India). He graduated from Govt. College of Engineering, Aurangabad affiliated with Dr. Babasaheb Ambedkar Marathwada University, Aurangabad and completed his postgraduate studies at Govt. College of Engineering Pune, affiliated to University of Pune. $\mathrm{He}$ is $\mathrm{Ph}$. D. in Computer Engineering at Shri Guru Gobind Singhji Institute of Engineering and Technology, Nanded, affiliated to Swami Ramanand Teerth Marathwada University Nanded (India).

Dr. Bichkar R. S. is currently working as Professor Department of E\&TC and Dean (R\&D), at G. H. Raisoni College of Engineering and Management, Wagholi, Pune (India). $\mathrm{He}$ is $\mathrm{Ph}$. D. from Indian Institute of Technology Kharagpur (India). His areas of research include the application of Genetic Algorithms to Image Processing and Data Mining. 